\title{
Buckling cascades in free sheets
}

\section{Wavy leaves may not depend only on their genes to make their edges crinkle.}

T he edge of a torn plastic sheet forms a complex three-dimensional fractal shape. We have found that the shape results from a simple elongation of the sheet in the direction along its edge. Natural growth processes in some leaves, flowers and vesicles could lead to a similar elongation and hence to the generation of characteristic wavy shapes.

We used rectangular plastic sheets pulled from the sides (in the $y$-direction) to generate a steadily travelling crack (in the $x$ direction). The high stresses near the crack tip produce an irreversible plastic deformation of the sheet and, as they are relieved, the deformed sheet is free to relax and to adopt a new shape in space.

Surprisingly, the equilibrium shape of the sheet consists of a cascade of waves upon waves along the newly formed edge. The waveform along the edge examined at six levels of magnification is self-similar, with a scaling factor of 3.2 (Fig. 1a). The amplitudes, $A$, of the waves in the cascades are simply related to their wavelengths $\lambda$ by $A=0.15 \lambda$ (Fig. 1b); the edge of the sheet is therefore a fractal. The fractal scaling spans 2.5 orders of magnitude and stops at a small length scale that is 6.5 times the sheet's thickness. Measurements of the deformation field revealed an increase in the length of the sheet in the $x$-direction that depends only on $y$, and decays smoothly to zero over a distance of a few millimetres from the edge (Fig. 1c).

How can such featureless deformations lead to such complex shapes? The key to this is to realize that the bending rigidity of thin plates is much smaller than their stretching rigidity ${ }^{1}$. Thus, to reduce its elastic energy, the sheet can easily buckle into shapes that remove in-plane compression. The thinner the sheet and the larger the compression, the smaller are the possible wavelengths of the buckles. This principle is seen in action during the crumpling of paper sheets $\mathrm{s}^{2-5}$ and the blistering of thin films $\mathrm{s}^{6,7}$, where sheets adopt very complex shapes in response to a uniform external loading.

Unlike these examples, our sheets (after tearing is complete) are free and not subjected to any external loading. The spontaneous buckling occurs as a consequence of the stresses created by the differential deformation within the sheet. Far from the edge, the deformation is small (Fig. 1c), and the compression leads to buckling that has a single long wavelength. Closer to the edge, the compression increases rapidly, leading to an ordered buckling sequence with smaller and smaller wavelengths.

This phenomenon should be general,
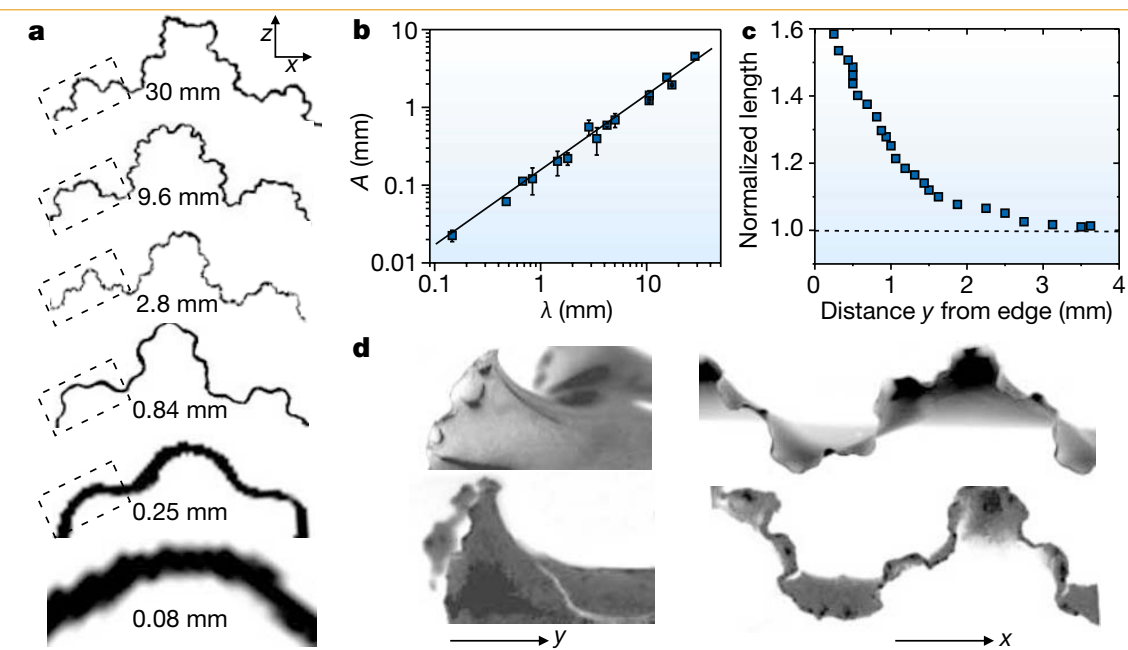

Figure 1 Buckling cascade in a deformed plastic sheet. a, Different magnifications of the edge of the sheet (0.012 mm thick). Successive pictures show the dotted boxed region on the left of the previous picture magnified by 3.2; the width of each image is indicated b, The amplitude of a wave in the cascade is related to its wavelength by $A=0.15 \lambda$ (solid line), for sheet thicknesses ranging from 0.012 to $0.5 \mathrm{~mm}$. c, The length of a sheet $(0.2 \mathrm{~mm}$ thick) in the $x$-direction, normalized by its original length, as a function of distance from the edge. Dashed line indicates the original length. $\mathbf{d}$, Side (left) and front (right) views of a plastic sheet (top panels) and a beet leaf (bottom panels), showing the similarity between the two buckled sheets.

and indeed we found buckling cascades in numerical investigations of elastic sheets with deformations similar to those shown in Fig. 1c. Although in the experiment the sheet thinned near the edge (owing to volume conservation), the simulations both with and without thinning revealed buckling cascades. In addition, numerical analysis indicates that the particular scaling properties of the buckling depend on the deformation profile.

A similar increase in length near an edge, and therefore similar internal stresses, can result from growth processes. The wavy structures found in many flower petals, lichens and leaves, such as the beet leaf in Fig. 1d, might be formed in this way. No genetic coding is needed to instruct pieces of a leaf to curl up and curl down. All that is required is a growth process to elongate the sheet along its edge - elasticity takes

care of the rest.

Eran Sharon*, Benoît Roman*, Michael Marder ${ }^{\star}$, Gyu-Seung Shin ${ }^{\star} \dagger$, Harry L. Swinney*

${ }^{\star}$ Center for Nonlinear Dynamics and Department of Physics, The University of Texas at Austin,

Austin, Texas 78712, USA

e-mail:eran@chaos.ph.utexas.edu

$\dagger$ Permanent address: Department of Physics, Kyung Hee University, Yongin, Kyunggi, Korea

1. Lord Rayleigh Theory of Sound Vol. 1, 396 (Dover, New York, 1945)

2. Ben Amar, M. \& Pomeau, Y. Proc. R. Soc. Lond. A 453, 729-755 (1997).

3. DiDonna, B. A., Witten, T. A., Venkataramani, S. C. \& Kramer, E. M. Phys. Rev. E 65, 016603-1-016603-25 (2002). 4. Matan, K., Williams, R., Witten, T. \& Nagel, S. Phys. Rev. Lett. 88, 076101-1-076101-4 (2002).

5. Kramer, E. \& Witten, T. Phys. Rev. Lett. 78, 1303-1306 (1997).

6. Argon, A., Gupta, V., Landis, H. \& Cornie, J. J. Mater. Sci. 24, 1207-1218 (1989)

7. Gioia, G. \& Ortiz, M. Adv. Appl. Mech. 33, 119-192 (1997). Competing financial interests: declared none.

\section{Thin films \\ Wrinkling of an elastic sheet under tension}

ere we consider the wrinkling of a stretched, slender elastic sheet and derive scaling laws for the wrinkle wavelength and amplitude that are valid far from the onset of buckling. Our results, which can be generalized to stretched and sheared anisotropic and inelastic sheets, could form the basis of a sensitive assay for the mechanical characterization of thin solid films.

When such a thin isotropic elastic sheet of thickness $t$, width $W$ and length $L$ $(t<<<L)$, composed of a material with Young's modulus $E$ and Poisson's ratio $\nu$, is subjected to a longitudinal stretching strain, $\gamma$, in its plane, it remains flat for $\gamma<\gamma_{c}$, a critical stretching strain. Further stretching causes the sheet to wrinkle (Fig. 1a). 

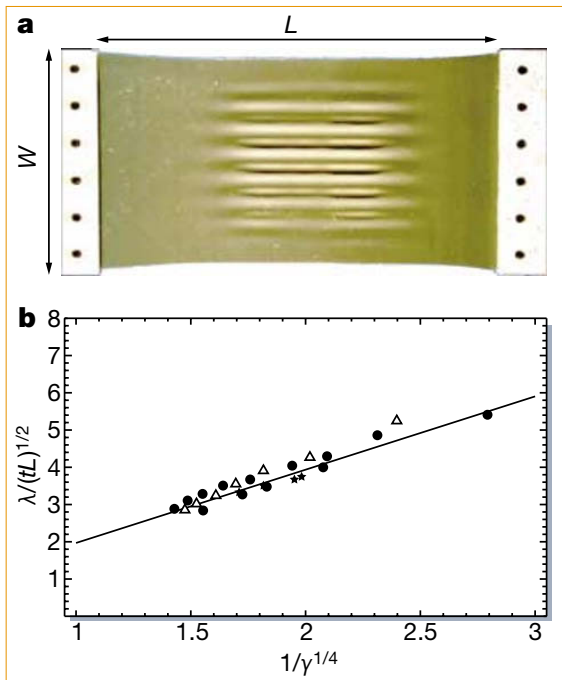

Figure 1 Wrinkles in a polyethylene sheet. a, Photograph of a sheet (length, $L, 20 \mathrm{~cm}$; width, $W, 12 \mathrm{~cm}$; thickness, $t, 0.01 \mathrm{~cm}$ ) under a uniaxial tensile strain, $\gamma \approx 0.1$. $\mathbf{b}$, Plot of the dimensionless wavelength $\lambda /(t L)^{1 / 2}$ against $1 / \gamma^{1 / 4}$ for various lengths of a polyethylene sheet. Lengths: circles, $25.4 \mathrm{~cm}$; stars, $30.4 \mathrm{~cm}$; triangles, $34.5 \mathrm{~cm}$. The data span more than an order of magnitude in strain, with $\gamma \in[0.01,0.2]$. The line corresponds to the theoretical prediction $\lambda /(t L)^{1 / 2}=\left(4 \pi^{2} / 3 \gamma\left(1-\nu^{2}\right)\right)^{1 / 4}$. For polyethylene, $\nu \approx 0.35$, so that $\lambda /(t L)^{1 / 2} \approx 2 / \gamma^{1 / 4}$, which is consistent with the results of our experiments.

This non-intuitive phenomenon occurs because the clamped boundaries prevent the sheet from contracting laterally in their vicinity, setting up a local biaxial state of stress; that is, the sheet is sheared near to its boundaries. The transverse stress is tensile near the clamped boundary and compressive slightly further from it ${ }^{1}$. When $\gamma \approx \gamma_{c}$, the sheet buckles to accommodate the in-plane strain incompatibility generated by the Poisson effect. However, typically $\gamma>\gamma_{c}$, so a linear theory is of little use and we must consider the geometrically nonlinear behaviour of the wrinkles.

For a sheet thus stretched, a periodic texture of parallel wrinkles of wavelength $\lambda<W$ decorates most of the sheet. The wavelength and amplitude are determined by minimizing the energy, $U=U_{\mathrm{B}}+U_{\mathrm{S}}$, where $U_{\mathrm{B}}$ is the energy due to bending (primarily in the transverse direction) and $U_{\mathrm{S}}$ is the energy due to stretching (along the wrinkles), subject to any geometric constraints (transverse inextensibility in this case).

If $A$ is the amplitude of the wrinkles, then $\quad U_{\mathrm{B}} \approx E t^{3}\left(A / \lambda^{2}\right)^{2} L W, \quad$ whereas $U_{\mathrm{S}} \approx \operatorname{Et} \gamma(A / L)^{2} L W$. Transverse inextensibility implies that $(A / \lambda)^{2} \approx \nu \gamma$, which quantifies the shrinkage of the sheet induced by the Poisson effect due to the imposed longitudinal strain. From this last relationship, we may derive $U \approx\left(E t^{3} / \lambda^{2}+E t \gamma \lambda^{2} / L^{2}\right) \nu \gamma L W$. Minimizing $U$ with respect to $\lambda$ gives a scaling law for the wavelength

$$
\lambda \approx(t L)^{1 / 2} / \gamma^{1 / 4}
$$

Substituting this relationship into the transverse-inextensibility constraint gives a scaling law for the amplitude, $A \approx(\nu t L)^{1 / 2} \gamma^{1 / 4}$. A refined calculation (E.C. and L.M., manuscript in preparation) gives the following expressions for the pre-factors: $C_{\lambda}=\lambda \gamma^{1 / 4} /(t L)^{1 / 2}=\left(4 \pi^{2} / 3\left(1-\nu^{2}\right)\right)^{1 / 4}$, and $C_{A}=A /(\nu t L)^{1 / 2} \gamma^{1 / 4}=\left(16 / 3 \pi^{2}\left(1-\nu^{2}\right)\right)^{1 / 4}$.

To verify our results, we stretched different lengths of a polyethylene sheet of thickness $\sim 0.01 \mathrm{~cm}$ and width $12 \mathrm{~cm}$, by using extensional strains $\gamma \in[0.01,0.2]$. The clamped boundary conditions were enforced by using two aluminium plates to sandwich the sheet, attached with doublesided adhesive tape to ensure that no slippage occurred. In Fig. 1b, we plot the experimental values of $\lambda /(t L)^{2}$ against $1 / \gamma^{1 / 4}$, together with our theoretical prediction, which shows quantitative agreement.

Our results complement those of classical tension-field theory ${ }^{2,3}$, which focuses on the much simpler problem of determining the locations of the wrinkles, but not their fine structure. The physics that underpins our calculation is as follows: a geometric packing constraint leads to the formation of wrinkles of intermediate wavelength because the bending resistance of the sheet penalizes short wavelengths and longitudinal stretching penalizes long wavelengths. Indeed, there is a mathematical similarity between the effect of the anisotropic tension on a curved surface and a simple elastic foundation, which allows us to generalize our results to a variety of applications (E.C. and L.M., manuscript in preparation). Moreover, inverting equation (1) yields $\gamma \approx t^{2} L^{2} / \lambda^{4}$, showing that wavelength is an extremely sensitive assay of strain in a wrinkled sheet.

Our results could form the basis of a quantitative wrinkling assay of thin solid films, and can potentially be applied to cell motility ${ }^{4}$, skin biomechanics and the mechanical characterization of soft solid membranes.

\section{E. Cerda ${ }^{\star} \dagger$, K. Ravi-Chandar $\ddagger$,}

L. Mahadevan ${ }^{\star}$

${ }^{\star}$ Department of Applied Mathematics and

Theoretical Physics, University of Cambridge,

Cambridge CB3 9EW, UK

e-mail:1.mahadevan@damtp.cam.ac.uk

$\dagger$ Departamento Fisica,

Universidad de Santiago de Chile,

Santiago, Chile

\$Department of Aerospace Engineering and

Engineering Mechanics, University of Texas, Austin, Texas 78705, USA

1. Benthem, J. Q. J. Mech. Appl. Math. 16, 413-430 (1963).

2. Wagner, H. Z. Flugtech. Motorluftschiffart Vol. 20, no. 8-12 (1929).

3. Mansfield, E. Proc. XII Int. Congr. Theor. Appl. Mech. (Springer, New York, 1968).

4. Harris, A. K., Wild, P. \& Stopak, D. Science 208, 177-179 (1980).

Competing financial interests: declared none.
Anthropogenic aerosols

\section{Indirect warming effect from dispersion forcing}

1 nthropogenic aerosols enhance cloud reflectivity by increasing the number concentration of cloud droplets, leading to a cooling effect on climate that is referred to as the Twomey effect ${ }^{1,2}$. Here we show that anthropogenic aerosols exert an additional effect on cloud properties that is derived from changes in the spectral shape of the size distribution of cloud droplets in polluted air and acts to diminish this cooling. This finding could help to improve our understanding of the indirect aerosol effect and its treatment in climate modelling.

An equation commonly used for examination of the indirect aerosol effect is

$$
r_{\mathrm{e}}=\beta[3 /(4 \pi \rho) L / N]^{1 / 3}
$$

where $\rho$ is the water density, $r_{\mathrm{e}}$ is the effective radius, $L$ is the cloud liquid-water content and $N$ is the number concentration of cloud droplets. The parameter $\beta$ is an increasing function of the relative dispersion $(\varepsilon)$ of the cloud droplet size distribution (ratio of the standard deviation to the mean radius), which is well described ${ }^{3,4}$ by

$$
\beta=\left(1+2 \varepsilon^{2}\right)^{2 / 3} /\left(1+\varepsilon^{2}\right)^{1 / 3}
$$

An increase (decrease) in effective radius causes a decrease (increase) in cloud reflectivity $^{1,2}$. A prevailing assumption implicit in the evaluation of the indirect aerosol effect is that increasing aerosol loading does not alter $\varepsilon$ or, hence, $\beta$. However, examination of data from field studies of the indirect aerosol effect shows that marine clouds classified as being polluted or having a continental origin generally have not only a larger $N$, but also a larger $\varepsilon$ relative to unaffected marine clouds.

Figure 1 shows the dependence of $\varepsilon$ and $\beta$ on $N$. The points connected by lines represent cases identified by different investigators as evidence for the indirect effect. In each case, the points with lower $N$ were characterized as background clouds and the higher points were characterized as similar clouds that had been perturbed by anthropogenic aerosols. Eleven of the 13 cases show an increase in $\varepsilon$ that is concurrent with an increase in $N$, with negligible change to slight decreases in the other two; there is also a general increase in $\varepsilon$ with $N$, as implied previously ${ }^{5-7}$.

One explanation for the simultaneous increase in $\varepsilon$ and $N$ is that anthropogenic aerosols have a more complex chemical composition and a broader size distribution than marine aerosols, and that the more numerous small droplets formed in a 